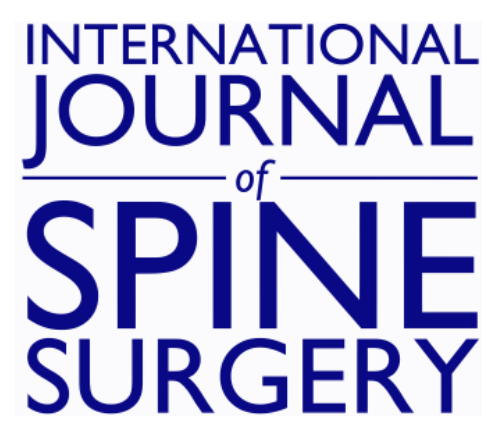

\title{
The Ankle-Pelvic Angle (APA) and Global Lower Extremity Angle (GLA): Summary Measurements of Pelvic and Lower Extremity Compensation
}

Max Vaynrub, Jared Tishelman, Aaron J. Buckland, Thomas J. Errico and Themistocles S.

Protopsaltis

Int J Spine Surg 2021, 15 (1) 130-136

doi: https://doi.org/10.14444/8017

http://ijssurgery.com/content/15/1/130

This information is current as of April 26, 2023.

Email Alerts Receive free email-alerts when new articles cite this article. Sign up at: http://ijssurgery.com/alerts 


\title{
The Ankle-Pelvic Angle (APA) and Global Lower Extremity Angle (GLA): Summary Measurements of Pelvic and Lower Extremity Compensation
}

\author{
MAX VAYNRUB, MD, JARED TISHELMAN, BA, AARON J. BUCKLAND, MD, THOMAS J. ERRICO, MD, \\ THEMISTOCLES S. PROTOPSALTIS, MD \\ Hospital for Joint Diseases at NYU Langone Medical Center, New York, New York
}

\begin{abstract}
Background: Adult sagittal spinal deformity (SSD) leads to the recruitment of compensatory mechanisms to maintain standing balance. After regional spinal compensation is exhausted, lower extremity compensation is recruited. Knee flexion, ankle flexion, and sacrofemoral angle increase to drive pelvic shift posterior and increase pelvic tilt. We aim to describe 2 summary angles termed ankle-pelvic angle (APA) and global lower extremity angle (GLA) that incorporate all aspects of lower extremity and pelvic compensation in a comprehensive measurement that can simplify radiographic analysis.

Methods: Full-body sagittal stereotactic radiographs were retrospectively collected and digitally analyzed. Spinal and lower extremity alignment were quantified with existing measures. Two angles-APA and GLA — were drawn as geometrically complementary angles to T1-pelvic angle (TPA) and global sagittal axis (GSA), respectively. Regression analysis was used to represent the predictive relationship between TPA and APA and between GSA and GLA.

Results: A total of 518 propensity score-matched patient records were available for analysis. Patients with lower extremity compensation had higher APA $\left(21.83^{\circ}\right.$ versus $\left.19.47^{\circ}, P=.007\right)$ and GLA $\left(6.03^{\circ}\right.$ versus $\left.1.19^{\circ}, P<.001\right)$ than those without compensation. APA and GLA demonstrated strong correlation with TPA $(r=0.81)$ and GSA $(r=0.77)$, respectively. Furthermore, the change between preoperative and postoperative values were strongly correlative between $\triangle \mathrm{APA}$ and $\triangle \mathrm{TPA}(r=0.71)$ and between $\triangle \mathrm{GLA}$ and $\triangle \mathrm{GSA}(r=0.77)$. APA above $20.6^{\circ}$ and GLA above $3.6^{\circ}$ were indicative of lower extremity compensation. Patients with increased GLA values had significantly higher Oswestry Disability Index scores (48.67 versus $41.04, P=.005$ ).

Conclusions: TPA and GSA are measures of global spinal alignment and APA and GLA, respectively, and are geometrically complementary angles that vary proportionately to SSD and balance the body. APA and GLA increase in SSD patients with lower extremity compensation and decrease with corrective surgery.

Level of Evidence: 4.

Clinical Relevance: APA and GLA offer a concise and simple method of communicating pelvic and lower extremity compensation.

Biomechanics

Keywords: ankle pelvic angle, global lower extremity angle, T1-pelvic angle, global sagittal axis, sagittal, deformity
\end{abstract}

\section{INTRODUCTION}

Adult sagittal spinal deformity (SSD) has become widely recognized as a significant cause of chronic pain and disability. ${ }^{1,2}$ Maximal efficiency of energy expenditure during standing posture is attained when the body is maintained within Dubousset's ${ }^{3}$ conus of economy. As spinal alignment shifts away from neutral, compensatory mechanisms are recruited to maintain maximally efficient upright balance. Initial stages of compensation involve reduction of thoracic kyphosis and increase of spinofemoral angle (SFA) and pelvic tilt (PT). The contribution of these regions to angular correction has anatomic limitations dictated by the ligamentous, capsular, and arthrodial constraints of the thoracic spine and hip joints, as well as atrophy or weakness of the muscles responsible for maintaining compensatory $\mathrm{PT}^{4}$ As the patient ages, these constraints become stiffer, leading to earlier exhaustion of their limits of compensation. ${ }^{5}$

Once the degree of malalignment exceeds the corrective limits of these mechanisms, the lower extremities are preferentially recruited for further compensation. Knee flexion (KA) and ankle flexion (AA) increase to drive the pelvic shift (PSh) further 
posterior. Surgical correction of the thoracolumbar deformity has been shown to reverse these compensatory changes. ${ }^{6}$

Whole body standing stereotactic radiography (STR) with the EOS (EOS Imaging, Paris, France) system provides an accurate assessment of spinopelvic and lower extremity parameters in the functional standing position. ${ }^{7-9}$ Obeid et $\mathrm{al}^{10}$ reviewed the STR images of 28 patients with SSD and demonstrated a strong correlation between increasing KA and lack of ideal lumbar lordosis as predicted with the formula proposed by Schwab et al. ${ }^{11}$ All patients with at least $10^{\circ}$ of KA lacked more than $30^{\circ}$ of lumbar lordosis. Lafage et $\mathrm{al}^{12}$ reviewed an STR database and demonstrated that, as SSD increases, KA increasingly contributes to increased pelvic retroversion and posterior $\mathrm{PSh}^{5} \mathrm{AA}$ and sacrofemoral angle are also used to describe lower extremity compensation, although the interaction among these parameters to determine final global balance is complex.

Given the growing variety of parameters used to describe various regions of spinal deformity, there is increased utility for comprehensive descriptors that summarize global balance. The T1-pelvic angle (TPA) quantifies SSD in a single angular measurement. ${ }^{13,14}$ Similarly, the global sagittal axis (GSA) measures full-body sagittal balance, including spinal, pelvic, and lower extremity contributions. ${ }^{15}$ Maintenance of upright posture dictates that, for each of these angular measurements, there exists a geometrically complementary angle that serves to maintain the center of gravity over the feet.

We aim to describe 2 summary lower extremity angles - the ankle-pelvic angle (APA) and the global lower extremity angle (GLA) - that are respectively complementary to TPA and GSA and incorporate all aspects of lower extremity compensation in a simplified form. We further aim to demonstrate their correlation with SSD and to identify cutoff values that indicate the presence of compensation.

\section{MATERIALS AND METHODS}

We tested the hypotheses that the summary angles APA and GLA (1) correlate with SSD, (2) correlate with surgical correction of SSD, (3) can identify the presence of pelvic and lower extremity deformity, and (4) correlate with functional outcome measures. A retrospective review was performed of a prospectively collected database of patients presenting with a primary spine complaint and undergoing full-body radiographic imaging at a single institution from 2013 to 2016. Included patients underwent preoperative or postoperative full-body STR imaging (EOS Imaging, Paris, France) or both. Subjects who underwent spinal corrective surgery had preoperative and postoperative films analyzed separately. Patients with a history of knee arthroplasty were excluded. Radiographic landmarks of interest were digitally identified using the validated surgical planning software Surgimap (Version 2.2.1, Nemaris Inc, New York, NY). Angular and linear measurements were then calculated.

Spinal sagittal alignment was described using TPA and GSA. Pelvic and lower extremity compensation were quantified using PT and KA. Comprehensive pelvic and lower extremity compensation were captured using APA and GLA. APA was defined as the angle between a line drawn from the bicoxofemoral axis to the midpoint of the sacral endplate and then from there to the ankle bimalleolar axis [Figure 1(left panel)]. GLA was defined as the angle between a line drawn from the distal femoral bicondylar axis to the midpoint of the sacral endplate and then from there to the ankle bimalleolar axis [Figure 1(right panel)].

The Oswestry Disability Index (ODI) was used to characterize disability at baseline and follow up. Based on an analysis of patients without SSD, we defined our threshold for the presence of lower extremity compensation as $\mathrm{KA}>6.9^{\circ}$.

\section{Statistical Methods}

Patients were propensity score matched to control for differences in age, gender, and body mass index (BMI) between the lower extremity compensation groups. Independent samples $t$ tests and paired $t$ tests were used for group comparisons, and bivariate correlations were characterized by Pearson correlation coefficients. Linear regression analysis was used to investigate the relationship between TPA and APA and between GSA and GLA.

\section{RESULTS}

A total of 1092 patients in the radiographic database were analyzed. After propensity score matching, 518 patient records were available for analysis, with 259 patients in the compensated and uncompensated groups, respectively. Here, 55.3\% 

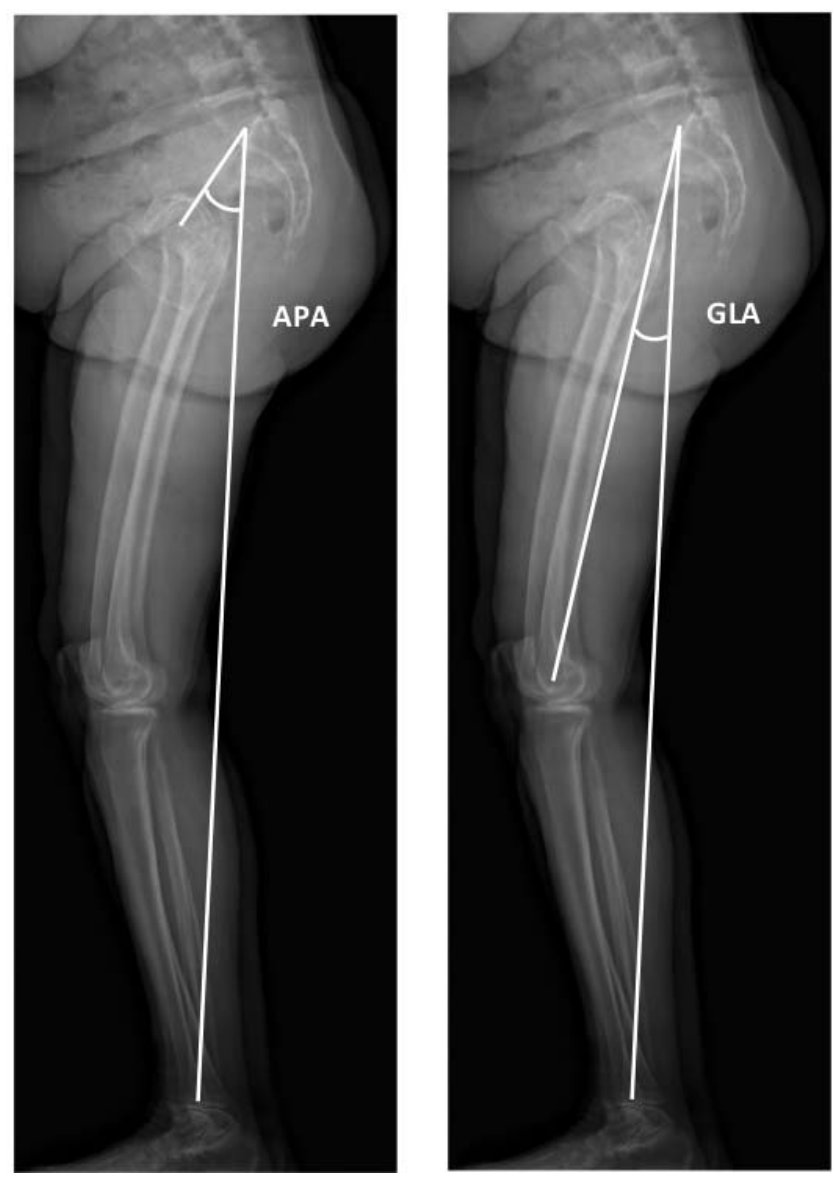

Figure 1. Ankle pelvic angle (APA) is defined as the angle between a line drawn from the bicoxofemoral axis to the midpoint of the sacral endplate and then from there to the ankle bimalleolar axis (left panel). Global lower extremity angle (GLA) is defined as the angle between a line drawn from the distal femoral bicondylar axis to the midpoint of the sacral endplate and then from there to the ankle bimalleolar axis (right panel).

of the cohort was female, the mean age was 58.41 years, and the mean BMI was 27.97. No significant difference existed in age, gender, or BMI between the cohorts with and without compensation. Mean KA was $13.4^{\circ}$ in patients with lower extremity compensation and $0.63^{\circ}$ in patients without $(P<$ .001 ; Table 1). APA values ranged from $-12.98^{\circ}$ to $35.44^{\circ}$. GLA values ranged from $-4.85^{\circ}$ to $13.24^{\circ}$.

Preoperative measurements demonstrated a significantly higher mean APA in patients with lower extremity compensation than those without $\left(21.83^{\circ}\right.$

Table 1. Preoperative demographic values.

\begin{tabular}{lcccr}
\hline & Whole Cohort & Low KA & High KA & P Value \\
\hline No. & 518 & 259 & 259 & NA \\
Mean KA, ${ }^{\circ}$ & $7.02 \pm 7.89$ & $0.63 \pm 4.07$ & $13.40 \pm 5.12$ & $<.001$ \\
Age, y & $58.41 \pm 16.7$ & $59.90 \pm 15.0$ & $61.41 \pm 13.4$ & .228 \\
Gender, \%F & 55.3 & 54.8 & 54.1 & .930 \\
BMI & $27.97 \pm 5.79$ & $28.29 \pm 5.25$ & $27.84 \pm 5.57$ & .338 \\
\hline
\end{tabular}

Abbreviations: BMI, body mass index; F, female; KA, knee flexion angle, NA, not applicable.
Table 2. Preoperative alignment comparison between lower extremity compensation groups.

\begin{tabular}{lrcr}
\hline & Low KA, ${ }^{\circ}$ & High KA, ${ }^{\circ}$ & $\boldsymbol{P}$ Value \\
\hline GLA & $1.19 \pm 1.73$ & $6.03 \pm 2.31$ & $<.001$ \\
APA & $19.47 \pm 8.29$ & $21.83 \pm 11.23$ & .007 \\
TPA & $15.40 \pm 9.86$ & $25.77 \pm 14.44$ & $<.001$ \\
GSA & $2.31 \pm 3.50$ & $9.05 \pm 5.32$ & $<.001$ \\
\hline
\end{tabular}

Abbreviations: APA, ankle pelvic angle; GLA, global lower extremity angle; GSA, global sagittal axis; KA, knee flexion angle; TPA, T1-pelvic angle.

versus $\left.19.47^{\circ}, P=.007\right)$. Similarly, mean GLA was greater in patients with lower extremity compensation than those without $\left(6.03^{\circ}\right.$ versus $1.19^{\circ}, P<$ $.001)$. Patients with lower extremity compensation also had higher mean TPA $\left(25.77^{\circ}\right.$ versus $15.40^{\circ}, P$ $<.001)$ and mean GSA $\left(9.05^{\circ}\right.$ versus $2.31^{\circ}, P<$ .001 ; Table 2).

Linear regression analysis revealed a strong correlation between APA and TPA $(r=0.79)$. Moreover, this correlation was more robust than the correlation between KA and TPA $(r=0.47)$. Similarly, strong correlation appeared between GLA and GSA $(r=0.77)$, which was greater than the correlation between KA and GSA $(r=0.72$; Table 3).

Furthermore, a subgroup analysis of patients with SSD and lower extremity compensation demonstrated a strong correlation between APA and TPA $(r=0.81)$ and a moderate one between GLA and GSA $(r=0.62)$. These comparisons were stronger than the correlations between KA and TPA $(r=0.36)$ and between KA and GSA $(r=0.54)$. A subanalysis of patients that did not exhibit lower extremity compensation demonstrated a strong correlation between APA and TPA $(r=0.82)$ and a moderate one between GLA and GSA $(r=0.56)$. These comparisons were stronger than the correlations between KA and TPA $(r=0.18)$ and between KA and GSA $(r=0.44)$.

\section{Analysis of Effects of Deformity Correction}

Analysis of the change in parameters after corrective deformity surgery demonstrated that the

Table 3. Linear regression analysis demonstrating correlations between preoperative variables of spinal alignment and lower extremity compensation, represented as $r$ values.

\begin{tabular}{lccc}
\hline & Whole Cohort & Low KA & High KA \\
\hline APA : TPA & 0.79 & 0.82 & 0.81 \\
KA : TPA & 0.47 & 0.18 & 0.36 \\
GLA : GSA & 0.77 & 0.56 & 0.62 \\
KA : GSA & 0.72 & 0.44 & 0.54 \\
\hline
\end{tabular}

Abbreviations: APA, ankle pelvic angle; GLA, global lower extremity angle; GSA, global sagittal axis; KA, knee flexion angle; TPA, T1-pelvic angle. 


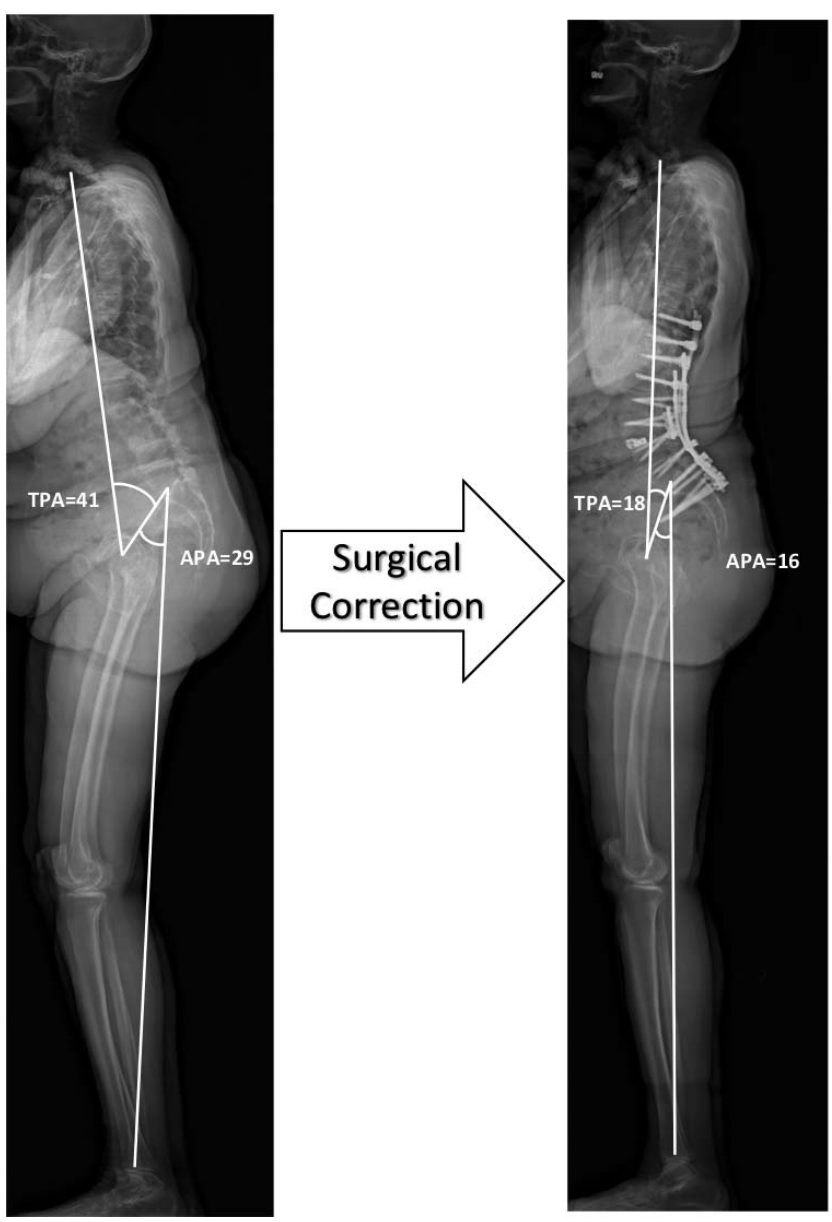

Figure 2. Preoperative (left panel) and postoperative (right panel) standing full-length radiographs of a patient with sagittal spinal deformity. Surgical correction of sagittal deformity results in decreased T1-pelvic angle (TPA), abatement of lower extremity compensation, and complementary decrease in ankle pelvic angle (APA).

above correlations remained significant in a dynamic setting (Figures 2 and 3). The change in APA between preoperative and postoperative values ( $\triangle \mathrm{APA})$ correlated strongly with the change in TPA ( $\triangle \mathrm{TPA} ; r=0.71)$. Similarly, the change in GLA between preoperative and postoperative values ( $\triangle$ GLA) correlated strongly with the change in GSA $(\Delta \mathrm{GSA} ; r=0.77)$.

Based on linear regression analysis, lower extremity compensation (defined as $\mathrm{KA}>6.9^{\circ}$ ) is present when an APA threshold of 20.6 is exceeded. Similarly, the threshold for GLA is $3.6^{\circ}$. We analyzed the difference in ODI between our cohorts using the above KA, APA, and GLA thresholds as determinants of lower extremity compensation. When stratified by the presence of compensation based on KA, patients with compensation had a significantly higher ODI than those without (48.73 versus $40.48, P=.002)$. Similarly, a significant

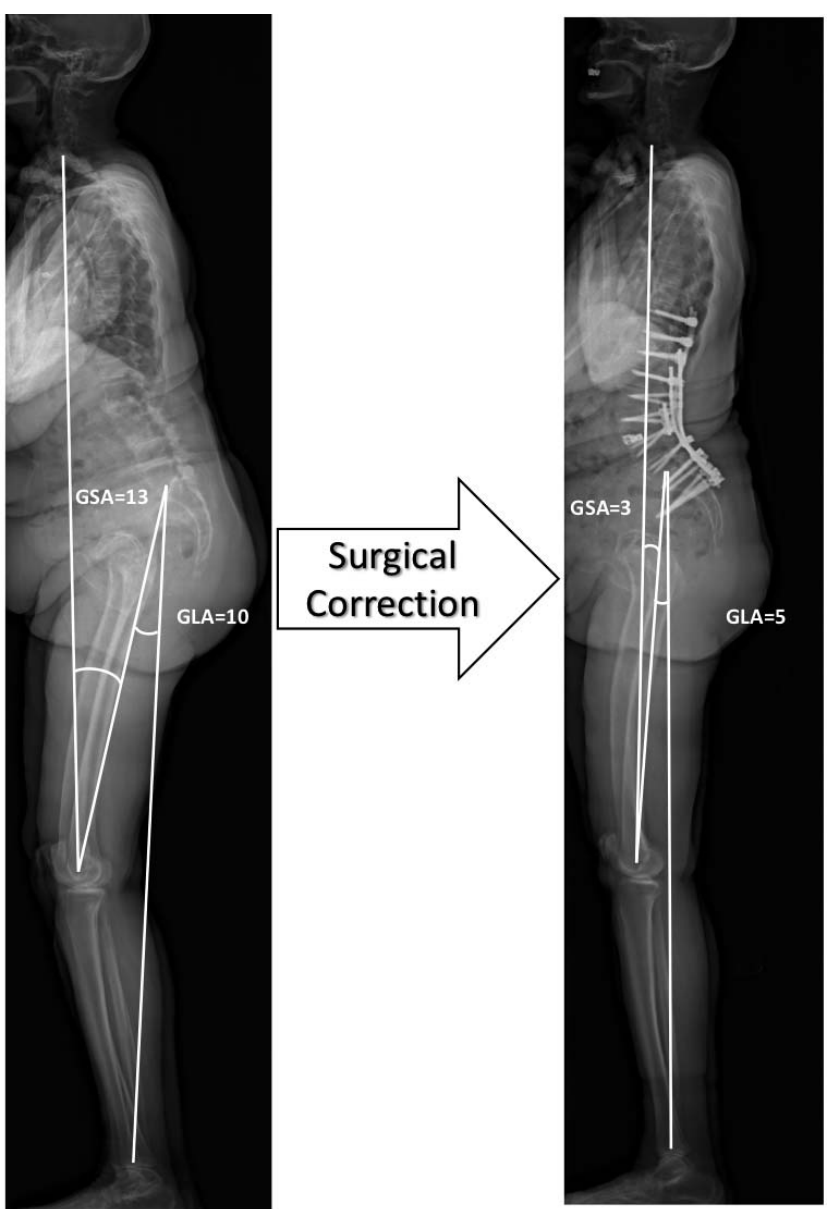

Figure 3. Preoperative (left panel) and (right panel) postoperative (right panel) standing full-length radiographs of a patient with sagittal spinal deformity. Surgical correction of sagittal deformity results in decreased global sagittal axis (GSA), abatement of lower extremity compensation, and complementary decrease in global lower extremity angle (GLA).

difference in ODI existed between patients with and without compensation as stratified by GLA (48.67 versus $41.04, P=.005)$. Stratification by APA also demonstrated a higher ODI in patients with compensation than those without, but the difference was nonsignificant (45.32 versus 43.22, $P=.445$; Table 4).

\section{DISCUSSION}

Compensatory mechanisms for SSD are triggered in order to align the spine over the pelvis, keeping it

Table 4. Oswestry Disability Index (ODI) scores for patients with and withou lower extremity compensation, as stratified by knee flexion angle (KA), ankle pelvic angle (APA), and global lower extremity angle (GLA).

\begin{tabular}{lccc}
\hline & Compensation Present & Compensation Absent & $\boldsymbol{P}$ Value \\
\hline KA & 48.73 & 40.48 & .002 \\
APA & 45.32 & 43.22 & .445 \\
GLA & 48.67 & 41.04 & .005 \\
\hline
\end{tabular}


within a conus of economy and minimizing the energy expenditure of staying upright. ${ }^{3}$ Itoi $^{16}$ performed one of the earliest full length radiographic analyses of lower extremity compensation in spinal deformity. He noted that incompletely compensated spinal deformity caused a compensatory retroversion of the pelvis, associated with hip extension, followed by KA with associated femoral inclination. More recently, Lafage et $\mathrm{al}^{12}$ described a predictive model of compensation which demonstrated that pelvic and lower extremity mechanisms are recruited in SSD to maintain the T9 vertebra aligned over the ankles. The multiple pelvic and lower extremity variables were shown to be correlated and predictive of the ultimate PT that allowed for alignment to be maintained. Therefore, it is not each angle's individual value that is significant but rather the overall effect on the position of the support structures below the spine.

While summary angles of spinal deformity (TPA and GSA) have been described and are commonly used in practice, pelvic and lower extremity compensation continues to be described in terms of individual but interrelated components (eg, PT, SFA, KA, AA). We define summary pelvic and lower extremity angles-APA and GLA - that enable upright balance by virtue of being geometrically complementary to TPA and GSA, respectively. We propose that the use of either APA or GLA can obviate the need for multiple lower extremity angular measurements, thus achieving a simpler and more intuitive description of pelvic and lower extremity compensation.

We have demonstrated that APA and GLA are both significantly increased in groups with lower extremity compensation compared with those without. Our definition of lower extremity compensation was based on KA, which showed significant correlation with TPA and GSA. The correlation of APA and GLA with TPA and GSA, respectively, was even stronger, implying a more direct relationship between these parameters than with KA. Furthermore, APA and GLA decreased after spinal corrective surgery in proportion to the decrease in TPA and GSA.

In addition to radiographic utility, the summary pelvic and lower extremity angles have clinical utility. ODI was significantly increased in patients with lower extremity compensation as detected by high GLA. Patients with high APA also had increased ODI, though the difference did not reach statistical significance. The thresholds of APA and GLA that define lower extremity compensation were calculated to be $20.6^{\circ}$ and $3.6^{\circ}$, respectively.

We chose TPA and GSA as descriptors of deformity because they describe global spinal alignment and have been shown to correlate with clinical outcome scores. ${ }^{15,17}$ TPA is readily attainable on most long-format radiographs. GSA requires full-length radiography including the lower extremities, attainable with the increasingly popular technology of full-body STR.

We chose KA as the defining variable for lower extremity compensation, as it is frequently used in clinical practice to radiographically identify the presence of compensation. A widely accepted threshold for the presence of compensation does not exist, though previous authors have mentioned values in the range of $2.5^{\circ}-10^{\circ} .{ }^{10,16}$ As intrinsic knee pathology can confound this analysis, we excluded patients with knee osteoarthritis. We then analyzed the mean KA in patients without SSD and chose a value that was 1 standard deviation higher than the mean as our threshold $\left(\mathrm{KA}>6.9^{\circ}\right)$.

There are several limitations to this study. Radiographic technique can be operator dependent and institution dependent. Our STR scans are obtained by protocol with the patient standing comfortably upright with hands on the clavicles. However, variations in technique may limit the generalizability of our reported thresholds at other institutions. A significant coincidence of lower extremity joint arthritis in the spinal deformity patient population may confound lower extremity angular measurements. Knee osteoarthritis can artificially increase KA, and its presence may negate the applicability of APA and GLA measurements. Hip osteoarthritis was not excluded from our study, as it contributes to overall sagittal malalignment. A subgroup analysis (results not reported) did not show any significant difference in results of patients with hip osteoarthritis. Lastly, most of our patients presented with some degree of sagittal plane deformity. Future studies should explore larger numbers of patients without deformity to further establish normative values and should examine an additional population of deformity patients for external validation. Additionally, prospective studies are needed to establish the clinical utility of either or both parameters as tools for decision making in the treatment of SSD. 


\section{CONCLUSIONS}

APA and GLA are singular measures combining pelvic and lower extremity compensation for SSD. TPA is a measure of global spinal alignment, and APA is a geometrically complementary angle that varies proportionately to SSD and balances the body in erect posture. Similarly, GLA accounts for compensation in a manner that is proportionate to the magnitude of spinal deformity defined by GSA. APA and GLA increase in SSD patients with lower extremity compensation and decrease with spinal corrective surgery. APA above $20.6^{\circ}$ and GLA above $3.6^{\circ}$ signal the presence of pelvic and lower extremity deformity.

All procedures performed in studies involving human participants were in accordance with the ethical standards of the institutional, national research committee, or both and with the 1964 Helsinki declaration and its later amendments or comparable ethical standards. For this type of study, formal consent is not required.

\section{REFERENCES}

1. Glassman SD, Berven S, Bridwell K, et al. Correlation of radiographic parameters and clinical symptoms in adult scoliosis. Spine (Phila Pa 1976). 2005;30(6):682-688.

2. Glassman SD, Bridwell K, Dimar JR, et al. The impact of positive sagittal balance in adult spinal deformity. Spine (Phila Pa 1976). 2005;30(18):2024-2029.

3. Dubousset J. Three-dimensional analysis of the scoliotic deformity. In: Weinstein SL, ed. The Pediatric Spine: Principles and Practice. New York, NY: Raven Press; 1994:479-496.

4. Lee CS, Lee CK, Kim YT, et al. Dynamic sagittal imbalance of the spine in degenerative flat back: significance of pelvic tilt in surgical treatment. Spine (Phila Pa 1976). 2001;26(18):2029-2035.

5. Diebo BG, Ferrero E, Lafage R, et al. Recruitment of compensatory mechanisms in sagittal spinal malalignment is age and regional deformity dependent: a full-standing axis analysis of key radiographical parameters. Spine (Phila Pa 1976). 2015;40(9):642-649.

6. Day LM, Ramchandran S, Jalai CM, et al. Thoracolumbar realignment surgery results in simultaneous reciprocal changes in lower extremities and cervical spine. Spine (Phila Pa 1976). 2017;42(11):799-807.

7. Deschenes S, Charron G, Beaudoin G, et al. Diagnostic imaging of spinal deformities: reducing patients radiation dose with a new slot-scanning x-ray imager. Spine (Phila Pa 1976). 2010;35(9):989-994.

8. Wybier M, Bossard P. Musculoskeletal imaging in progress: the EOS imaging system. Joint Bone Spine. 2013;80(3):238-243.

9. Ferrero E, Liabaud B, Challier V, et al. Role of pelvic translation and lower-extremity compensation to maintain gravity line position in spinal deformity. $J$ Neurosurg Spine. 2016;24(3):436-446.
10. Obeid I, Hauger O, Aunoble S, et al. Global analysis of sagittal spinal alignment in major deformities: correlation between lack of lumbar lordosis and flexion of the knee. Eur Spine J. 2011;20(Suppl 5):681-685.

11. Schwab F, Lafage V, Patel A, et al. Sagittal plane considerations and the pelvis in the adult patient. Spine (Phila Pa 1976). 2009;34(17):1828-1833.

12. Lafage R, Liabaud B, Diebo BG, et al. Defining the role of the lower limbs in compensating for sagittal malalignment. Spine (Phila Pa 1976). 2017;42(22):E1282-E1288.

13. Protopsaltis T, Schwab F, Bronsard N, et al. The T1 pelvic angle, a novel radiographic measure of global sagittal deformity, accounts for both spinal inclination and pelvic tilt and correlates with health-related quality of life. $J$ Bone Joint Surg Am. 2014;96(19):1631-1640.

14. Ryan DJ, Protopsaltis TS, Ames CP, et al. T1 pelvic angle (TPA) effectively evaluates sagittal deformity and assesses radiographical surgical outcomes longitudinally. Spine (Phila Pa 1976). 2014;39(15):1203-1210.

15. Diebo BG, Oren JH, Challier V, et al. Global sagittal axis: a step toward full-body assessment of sagittal plane deformity in the human body. $J$ Neurosurg Spine. 2016;25(4):494-499.

16. Itoi E. Roentgenographic analysis of posture in spinal osteoporotics. Spine (Phila Pa 1976). 1991;16(7):750-756.

17. Banno T, Hasegawa T, Yamato Y, et al. T1 pelvic angle is a useful parameter for postoperative evaluation in adult spinal deformity patients. Spine. 2016;41(21):1641-1648.

Disclosures and COI: The manuscript submitted does not contain information about medical device(s)/drug(s). No funds were received in support of this work. Dr Buckland reports personal fees from Stryker and Nuvasive outside the submitted work. Dr Errico reports personal fees from K2M, grants from Pfizer and ISSGF, and other from Fastenetix and Harms Study Group outside the submitted work. Dr Protopsaltis reports personal fees from Medicrea, NuVasive, Innovasis, and K2M; grants from Cervical Spine Research Society and Zimmer Biomet; and other from Torus Medical and Globus Medical outside the submitted work. Dr Vaynrub and Dr Tishelman have nothing to disclose. The authors report no conflict of interest concerning the materials or methods used in this study or the findings specified in this paper.

Portions of this work were presented in abstract form at North American Spine Society Annual Meeting, Orlando, FL, in October 2017 and in poster form at Scoliosis Research Society Annual Meeting, Philadelphia, PA, in September 2017.

Corresponding Author: Max Vaynrub, MD, Hospital for Joint Diseases at NYU Langone Medical Center, 1275 York Ave H-1011, New 
York, NY 10065. Phone: (212) 639-2000; Fax: (212) 717-3573; Email: Max.Vaynrub@gmail.com.

Published 26 February 2021

This manuscript is generously published free of charge by ISASS, the International Society for the Advancement of Spine Surgery. Copyright (C) 2021 ISASS. To see more or order reprints or permissions, see http://ijssurgery.com. 